\title{
The pattern of postoperative quality of life following minimally invasive gastrectomy for gastric cancer: a prospective cohort from Korean multicenter robotic gastrectomy trial
}

\author{
Jong-Ho Choi', Sang-Uk Han², Han-Kwang Yang ${ }^{1,3}$, Young-Woo Kim4, Keun Won Ryư ${ }^{4}$, Joong-Min Park ${ }^{5}$, \\ Ji Yeong An ${ }^{6}$, Min-Chan Kim ${ }^{7}$, Sungsoo Park ${ }^{8}$, Kyo Young Song ${ }^{9}$, Sung Jin $\mathrm{Oh}^{10}$, Seong-Ho Kong ${ }^{1}$, \\ Byoung Jo Suh ${ }^{10}$, Dae Hyun Yang ${ }^{11}$, Tae Kyung Ha ${ }^{12}$, Hyoung-II Kim ${ }^{6}$, Woo Jin Hyung ${ }^{6}$, Hyuk-Joon Lee ${ }^{1,3}$ \\ ${ }^{1}$ Department of Surgery, Seoul National University College of Medicine, Seoul, Korea \\ ${ }^{2}$ Department of Surgery, Ajou University College of Medicine, Suwon, Korea \\ ${ }^{3}$ Cancer Research Institute, Seoul National University College of Medicine, Seoul, Korea \\ ${ }^{4}$ Center for Gastric Cancer, National Cancer Center, Goyang, Korea \\ ${ }^{5}$ Department of Surgery, Chung-Ang University College of Medicine, Seoul, Korea \\ ${ }^{6}$ Department of Surgery, Yonsei University College of Medicine, Seoul, Korea \\ ${ }^{7}$ Department of Surgery, Dong-A University College of Medicine, Busan, Korea \\ ${ }^{8}$ Department of Surgery, Korea University College of Medicine, Seoul, Korea \\ ${ }^{9}$ Department of Surgery, College of Medicine, The Catholic University of Korea, Seoul, Korea \\ ${ }^{10}$ Department of Surgery, Inje University College of Medicine, Busan, Korea \\ ${ }^{11}$ Department of Surgery, Hallym University College of Medicine, Seoul, Korea \\ ${ }^{12}$ Department of Surgery, Hanyang University College of Medicine, Seoul, Korea
}

Purpose: Quality of life (QOL) has become important in the trend of emphasizing patient satisfaction. This study aimed to evaluate the QOL in patients who underwent laparoscopic or robotic gastrectomy for gastric cancer.

Methods: A prospective trial was performed involving patients who underwent laparoscopic or robotic gastrectomy for primary gastric cancer at 11 hospitals in Korea. Within this comparative trial, QOL, postoperative pain, and long-term complications were exanimated. The quality-of-life questionnaire (QLQ)-C30 and QLQ-STO22 developed by the European Organization for Research and Treatment of Cancer were used for the QOL survey. We compared the data after dividing it into several types of characteristics as follows; device (robotic or laparoscopic), operation type, pathological stage, and sex. Biased components were extracted by logistic regression analysis. Propensity score matching was applied to the data set with the biased components.

Results: In total, 434 patients (211 for laparoscopic surgery and 223 for robotic surgery) were enrolled, out of which 321 patients who responded to both preoperative and postoperative surveys were selected for analysis. Robotic gastrectomy was not different from laparoscopic gastrectomy with respect to postoperative QOL. Distal gastrectomy showed better scores than total gastrectomy in terms of role functioning, social functioning, fatigue, nausea/vomiting, pain, dyspnea, constipation, financial difficulties, dysphagia, eating restrictions, anxiety, taste, and body image. Male patients showed better scores on the 19 scales compared to female patients.

Conclusion: Robotic and laparoscopic approaches for gastric cancer surgery did not differ from each other with respect to QOL. Distal gastrectomy resulted in better QOL than total gastrectomy.

[Ann Surg Treat Res 2020;99(5):275-284]

Key Words: Gastrectomy, Quality of life, Stomach neoplasms

Received March 2, 2020, Revised July 13, 2020, Accepted August 11, 2020 Corresponding Author: Hyuk-Joon Lee

Department of Surgery, Seoul National University College of Medicine, 101 Daehak-ro, Jongno-gu, Seoul 03080, Korea

Tel: +82-2-2072-2318, Fax: +82-2-766-3975

E-mail: appe98@snu.ac.kr

ORCID: https://orcid.org/0000-0002-9530-647X
Copyright (c) 2020, the Korean Surgical Society

(c) Annals of Surgical Treatment and Research is an Open Access Journal. All articles are distributed under the terms of the Creative Commons Attribution NonCommercial License (http://creativecommons.org/licenses/by-nc/4.0/) which permits unrestricted non-commercial use, distribution, and reproduction in any medium, provided the original work is properly cited. 


\section{INTRODUCTION}

The discovery of minimally invasive surgical techniques has played an important role in the development of surgical treatment for gastric cancer. It is currently preferred over open surgery in early gastric cancer because of its low complication rate and enhanced surgical view [1]. Research concerning laparoscopic surgery has compared the oncological effects of surgical techniques through a series of randomized control trials and has found that minimally invasive surgery is not inferior to open surgery [2,3]. With the increasing survival rate of patients with gastric cancer, function preservation has become another key factor in gastric cancer surgery. Comparative trials of partial gastrectomy, including pylorus-preserving gastrectomy (PPG) or proximal gastrectomy $(\mathrm{PG})$, are underway regarding longterm surgical outcomes [4,5]. Studies on surgical resection margin and safety have continuously been carried out [6]. In an atmosphere that emphasizes patient satisfaction, quality of life (QOL) after surgery has become a topic of increasing focus.

Systemic indicators have been developed to evaluate QOL after surgery. The commonly used QOL evaluation tools for gastric cancer include the quality-of-life questionnaire (QLQ)-C30 and QLQ-STO22 developed by the European Organization for Research and Treatment of Cancer (EORTC), which has good reliability and validity [7]. The Korean version of the EORTC QLQ-C30 was validated in 2004 and has been widely used in postoperative patient surveys [8]. Various comparative studies have been conducted using these indicators for QOL [9].

Laparoscopic gastrectomy requires a high degree of experience in lymph node dissection. It takes a long time to train a skilled laparoscopic surgeon. Robotic surgery, a modification of conventional laparoscopic surgery, is an emerging solution for this difficult laparoscopic training [10]. Robotic surgery requires a relatively short training period with a systemic assistant, and provides benefits such as an easy surgical view, free joint movement, and handshake correction [11]. Currently, the number of robotic surgeries is increasing worldwide. Although they emphasize the advantages of robotic surgery, many studies have reported limitations regarding single-institute and retrospective data collection. In a recent, multicenter prospective trial study comparing robotic gastrectomy with laparoscopic gastrectomy, no significant difference in outcomes was found between these two operations regarding operative bleeding, open conversion rate, and hospital stay [12]. We further analyzed the QOL in this study using the same data set as that of this multicenter prospective trial.

\section{METHODS}

\section{Patients and treatment}

A prospective multicenter study was conducted on patients who underwent laparoscopic or robotic gastrectomy at 11 hospitals in Korea between May 2011 and December 2012. The type of operation (extent of gastrectomy) was determined by the patient after a comprehensive understanding of the location of the tumor, clinical stage, and operative procedure. Investigators generally followed the international gastric cancer guideline of the Japanese Gastric Cancer Association [13]. This study was performed by surgeons who participated in the Korean Laparoendoscopic Gastrointestinal Surgery Study Group, and the laparoscopic or robotic procedures were accordingly standardized in the multicenter. The robotic procedure was performed using the da Vinci Surgical System (Intuitive Surgical, Sunnyvale, CA, USA). The adjuvant chemotherapy regimen (capecitabine plus oxaliplatin or S-1) was recommended for patients with pathological stage II or III disease in both the groups [14,15]. We collected clinical information such as age, sex, weight, body mass index (BMI), American Society of Anesthesiologists (ASA) physical status classification, tumor location, pathological stage, operation type, and approach of the anastomosis, accordingly for each subject. Pathological evaluation and staging were determined according to the 7th American Joint Committee on Cancer staging system.

\section{Study design}

Since the patients who chose robotic surgery group were fewer in number, we registered one laparoscopic surgical subject after recruiting one robotic surgical subject with the same surgeon, sex, and extent of gastric resection. The inclusion criteria were as follows: (1) a documented diagnosis of gastric adenocarcinoma, (2) minimally invasive gastric cancer surgery, and (3) no preoperative evidence of serosal invasion or extraperigastric lymph node metastasis during the preoperative examination. Exclusion criteria were as follows: (1) mental incompetency, (2) illiteracy, (3) pregnancy, or (4) aged younger than 20 years. A paper survey was given at 11 centers. The Korean version of the EORTC QLQ-C30 and EORTC QLQSTO22 was used for the survey. The QOL questionnaire was collected at the time of preoperative admission, postoperative 1st outpatient department (OPD) visit (usually 2 weeks after discharge), followed by 3-month, 1-year, and 3-year OPD visits respectively, postsurgery. Patients who responded to 1 preoperative questionnaire and similarly gave their responses to at least one among the several questionnaires administered postoperatively were selected as the initial data set. We compared the data after dividing it into several types of characteristics as follows: device (robotic or laparoscopic), operation type (distal gastrectomy [DG], total gastrectomy [TG], 
or PPG), pathological stage (stage I or stage II-III), and sex (male or female). Each comparison used data set after propensity score matching.

\section{Propensity score matching}

In the comparative data set, biased components were extracted by logistic regression analysis. Propensity score matching (PSM) was applied to the initial data set by PSM tool in IBM SPSS Statistics ver. 25.0 (IBM Corp., Armonk, NY, USA) with biased components (confounders). Nearest-neighbor matching and 1:1 matching were used in the software as an option. Exceptionally, comparison by operation type did not adjust the bias according to the location of the tumor. The cases of PPG and PG were excluded from the initial data set during comparison of DG and TG. Similarly, for comparison of DG and PPG, the cases of TG and PG were excluded from the initial data set. Each comparison was performed using the respective logistic regression and PSM.

\section{Outcomes}

The primary outcome of this study was QOL after gastrectomy for gastric cancer. QLQ-C30 consists of 30 questions, which assess 15 scales; general health status, physical functioning, role functioning, emotional functioning, cognitive functioning, social functioning, fatigue, nausea/ vomiting, pain, dyspnea, insomnia, appetite loss, constipation, diarrhea, and financial difficulties. QLQ-STO22 consists of 22 questions, which assess 9 scales: body image, dysphagia, pain, reflux symptoms, eating restrictions, anxiety, dry mouth, taste, and hair loss. The secondary outcomes of this study were pain and long-term complications. Pain was assessed using the visual analog scale (VAS, 0-10) and therapeutic regimen for pain killer. The pain scale and the volume usage of patientcontrolled analgesia (PCA) were checked on the operation day, postoperative day (POD) 1 to POD 10, and discharge day. Intestinal obstruction, stenosis, reflux, malabsorption, dumping, and delayed emptying, which appeared after 1 month of surgery, were monitored as long-term complications. Student t-test, chi-square test, and mixed analysis of variance (ANOVA) were used for comparative statistical analyses. All statistical analyses were performed at a significance level of 5\% using IBM SPSS statistics.

\section{Ethical standards}

The study protocol was approved by the Institutional Review Boards of all participating institutions (No. H-1710050-891, Seoul National University Hospital). This multicenter prospective study was approved by the responsible committee on human experimentation of the respective medical center and all procedures followed the Helsinki Declaration of 1964 and later versions of the same guideline. All patients signed an informed consent form before the study.

\section{RESULTS}

\section{Demographics and propensity score matching}

In the multicenter project, 434 patients (robotic 223 and laparoscopic 211) were enrolled from 11 centers. During the 5 surveys, 337, 302, 157, 220, and 165 patients answered at the time of preoperative admission, postoperative 1st OPD visit, and 3 months, 1 year, and 3 years later, respectively (Fig. 1). Among them, 321 patients responded to both the pre- and postoperative surveys: 72 patients answered at 1 preoperative and 4 postoperative surveys; 95 patients answered at 1 preoperative and 3 postoperative surveys; 117 patients answered at 1 preoperative and 2 postoperative surveys; 37 patients answered 1 preoperative and 1 postoperative survey. We compared these 321 patients accordingly on the basis of the following groups: (1) robotic vs. laparoscopic, (2) TG vs. DG, (3) PPG vs. DG, (4) stage I vs. stage II-III, and (5) male vs. female. Comparing the 147 patients in the laparoscopic group and the 174 patients of the robotic group, there was a significant difference in age, ASA physical status classification, and pathologic TNM; the patients in the robotic group were young and had an advanced pathological stage (Table 1). We adjusted

\begin{tabular}{lc|c|c|c|c|}
\multirow{2}{*}{ A } & \multicolumn{1}{c}{$\# 1$} & \multicolumn{1}{c}{$\# 2$} & $\# 3$ & $\# 4$ & $\# 5$ \\
\cline { 2 - 7 } $\begin{array}{r}\text { Survey } \\
\text { schedule }\end{array}$ & Preoperative & 1st OPD & 3 Mo & 1 Yr & 3 Yr \\
\cline { 2 - 7 } Answer & 337 & 302 & 157 & 220 & 165
\end{tabular}

B

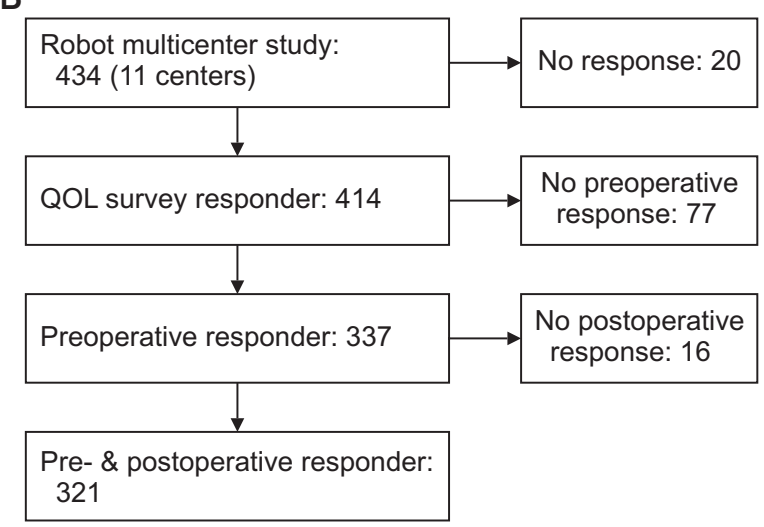

Fig. 1. Quality of life (QOL) survey. (A) The schedule of the QOL survey. Five surveys were completed at the time of preoperative admission, postoperative 1 st outpatient department (OPD) visit after discharge, and 3 months, 1 year, and 3 years. The answers were collected from 337, 302, 157, 220, and 165 patients at the respective time points. (B) The response of the survey. Robot multicenter study collected surveys from 434 patients from 11 centers, and 113 patients did not answer both preoperative and postoperative surveys. Accordingly, 321 cases were used as the initial data set for the following analyses. 
Table 1. Demographics of participants

\begin{tabular}{|c|c|c|c|c|c|c|}
\hline \multirow{2}{*}{ Characteristic } & \multicolumn{3}{|c|}{ Before PSM $(n=321)$} & \multicolumn{3}{|c|}{ After PSM ( $n=262)$} \\
\hline & $\begin{array}{l}\text { Laparoscopic } \\
\qquad(\mathrm{n}=147)\end{array}$ & $\begin{array}{l}\text { Robotic } \\
(n=174)\end{array}$ & P-value & $\begin{array}{l}\text { Laparoscopic } \\
\qquad(\mathrm{n}=131)\end{array}$ & $\begin{array}{l}\text { Robotic } \\
(n=131)\end{array}$ & P-value \\
\hline Age $(y r)$ & $55.3 \pm 11.1$ & $51.9 \pm 10.8$ & 0.005 & $54.8 \pm 10.8$ & $52.9 \pm 11.1$ & 0.151 \\
\hline Male sex & $87(59.2)$ & $103(59.2)$ & 0.998 & $77(58.8)$ & $79(60.3)$ & 0.801 \\
\hline Weight (kg) & $63.3 \pm 9.7$ & $64.2 \pm 11.3$ & 0.460 & $63.1 \pm 9.7$ & $64.6 \pm 11.3$ & 0.261 \\
\hline Body mass index $\left(\mathrm{kg} / \mathrm{m}^{2}\right)$ & $23.6 \pm 2.6$ & $23.6 \pm 3.0$ & 0.955 & $23.6 \pm 2.6$ & $23.8 \pm 3.0$ & 0.410 \\
\hline ASA PS classification & & & 0.036 & & & 0.841 \\
\hline 1 & $96(65.3)$ & $135(77.6)$ & & $93(71.0)$ & $93(71.0)$ & \\
\hline 2 & $50(34.0)$ & $37(21.3)$ & & $37(28.2)$ & $36(27.5)$ & \\
\hline 3 & $1(0.7)$ & $2(1.1)$ & & $1(0.8)$ & $2(1.5)$ & \\
\hline Tumor location & & & 0.446 & & & 0.528 \\
\hline Upper third & $14(9.5)$ & $26(14.9)$ & & $13(9.9)$ & $20(15.3)$ & \\
\hline Mid third & $55(37.4)$ & $66(37.9)$ & & $51(38.9)$ & $47(35.9)$ & \\
\hline Lower third & $77(52.4)$ & $79(45.4)$ & & $66(50.4)$ & $61(46.6)$ & \\
\hline Multiple & $1(0.7)$ & $2(1.1)$ & & $1(0.7)$ & $3(2.3)$ & \\
\hline pTNM stage & & & 0.020 & & & 0.298 \\
\hline $\mathrm{IA}$ & $125(85.0)$ & $116(66.7)$ & & $111(84.7)$ & $100(76.3)$ & \\
\hline IB & $10(6.8)$ & $21(12.1)$ & & $9(6.9)$ & $19(14.5)$ & \\
\hline IIA & $8(5.4)$ & $17(9.8)$ & & $7(5.3)$ & $4(3.1)$ & \\
\hline IIB & $2(1.4)$ & $10(5.7)$ & & $2(1.5)$ & $5(3.8)$ & \\
\hline IIIA & $1(0.7)$ & $6(3.4)$ & & $1(0.8)$ & $1(0.8)$ & \\
\hline IIIB & $0(0)$ & $3(1.7)$ & & $0(0)$ & $1(0.8)$ & \\
\hline IIIC & $1(0.7)$ & $1(0.6)$ & & $1(0.8)$ & $1(0.8)$ & \\
\hline Operation type & & & 0.709 & & & 0.875 \\
\hline DG & $113(76.9)$ & $124(71.3)$ & & $100(76.3)$ & $100(76.3)$ & \\
\hline TG & $22(15.0)$ & $32(18.4)$ & & $19(14.5)$ & $22(16.8)$ & \\
\hline PPG & $11(7.5)$ & $17(9.8)$ & & $11(8.4)$ & $8(6.1)$ & \\
\hline PG & $1(0.7)$ & $1(0.6)$ & & $1(0.8)$ & $1(0.8)$ & \\
\hline Approach of anastomosis & & & 0.412 & & & 0.778 \\
\hline Intracorporeal & $109(74.1)$ & $126(72.4)$ & & $96(73.3)$ & $98(74.8)$ & \\
\hline Extracorporeal & $38(25.9)$ & $48(27.6)$ & & $35(26.7)$ & $33(25.2)$ & \\
\hline
\end{tabular}

Values are presented as mean \pm standard deviation or number $(\%)$.

We used propensity score matching (PSM) to correct selection bias because the patient's demographics were heterogeneous.

ASA, American Society of Anesthesiologists; PS, physical status; pTNM, pathologic TNM; DG, distal gastrectomy; TG, total gastrectomy; PPG, pylorus-preserving gastrectomy; PG, proximal gastrectomy.

the 2 groups into 262 patients (robotic 131 vs. laparoscopic 131) by PSM. A total of $46.6 \%$ (robotic) and $50.4 \%$ (laparoscopic) of tumors located in the lower third of the stomach. A total of $76.3 \%$ (robotic) and $84.7 \%$ (laparoscopic) of patients were in stage IA. A total of $76.3 \%$ of operations required DG. Intracorporeal anastomosis was performed in $74.8 \%$ (robotic) and $73.3 \%$ (laparoscopic) of cases.

\section{Similar QOL between robotic and laparoscopic approaches}

Twenty-four scales composed of general health status, 5 of C30 functioning scales, 9 of C30 symptom scales, and 9 of STO22 symptom scales were checked to compare robotic and laparoscopic surgery. Only C30-cognitive functioning was significantly different between the 2 groups $(P=0.005$ ) (Fig.
2). The robotic group had better preoperative QOL in cognitive functioning than the laparoscopic group $(P=0.010)$ and the scale did not change over time $(P=0.053)$. General health status, physical functioning, and role functioning decreased in the immediate postoperative period and then recovered. The emotional and social functioning increased after the operation. Symptom scales, except for 2 scales, were increased and recovered serially after surgery; dyspnea and constipation scales did not change over time $(\mathrm{P}=0.086$ and $\mathrm{P}=0.263$, respectively).

\section{Long-term complications and pain scores}

Considering 6 major complications (intestinal obstruction, stenosis, reflux, malabsorption, dumping, and delayed emptying), the incidence of any type of complication was as 


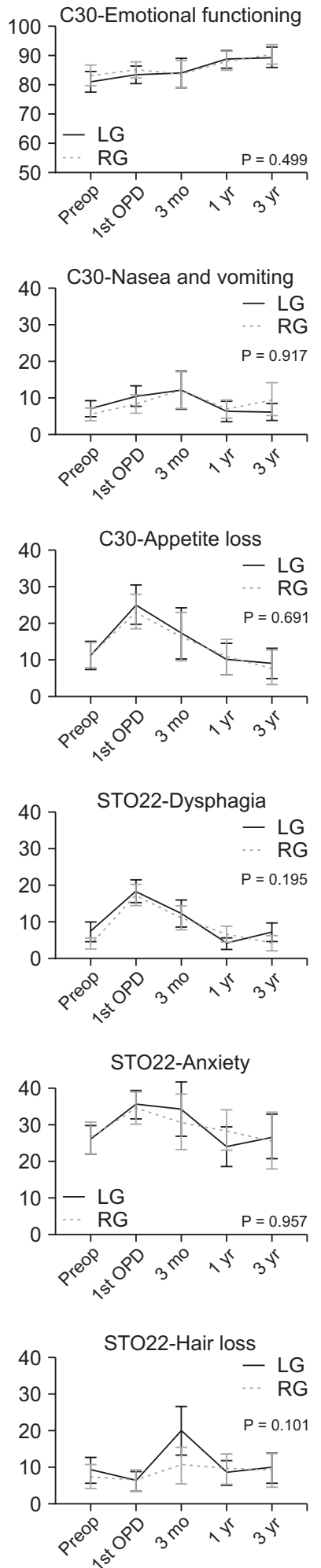

Fig. 2. Quality of life (QOL) of laparoscopic surgery vs. robotic surgery. The QOL questionnaire (QLQ)-C30 and QLQ-STO22 developed by the European Organization for Research and Treatment of Cancer were used. A total of 24 scales are presented in the graph. In the respective scale, the maximum score is 100 and the minimum score is 0 . A high score of general health status and 5 functioning scales indicates a good QOL. A high score of 18 symptom scales indicates a poor QOL. LG, laparoscopic gastrectomy; RG, robotic gastrectomy; Preop, preoperative; OPD, outpatient department. 
low as 9.2\% (24/262) within 3 years after discharge (Table 2). Laparoscopic or robotic approaches did not show a significant difference in long-term complications. For example, the rate of delayed emptying was higher in the laparoscopic group (3/131 vs. $0 / 131, P=0.861$ ) without significance. Postoperative pain was compared using 4 indexes (the VAS of resting pain, the VAS of movement pain, the frequency of additional painkillers, and the volume usage of PCA). The score of resting pain and movement pain decreased daily from the highest score on the operation day; however, the usage of painkillers and PCA volume did not change significantly after the operation. There was no significant difference in the 4 pain indices between laparoscopic and robotic group (resting pain sore, $\mathrm{P}=0.614$; movement pain score, $\mathrm{P}=0.421$; pain killer, $\mathrm{P}=0.824$; $\mathrm{PCA}$ usage, $P=0.524$ ) (Fig. 3).

Table 2. Long-term complications

\begin{tabular}{|c|c|c|c|c|c|c|c|c|c|c|c|}
\hline \multirow{2}{*}{ Complication } & \multicolumn{5}{|c|}{ Laparoscopic gastrectomy $(\mathrm{n}=131)$} & \multicolumn{5}{|c|}{ Robotic gastrectomy $(\mathrm{n}=131)$} & \multirow{2}{*}{ P-value } \\
\hline & $6 \mathrm{mo}$ & $1 \mathrm{yr}$ & $2 y r$ & $3 \mathrm{yr}$ & Total & $6 \mathrm{mo}$ & $1 \mathrm{yr}$ & $2 y r$ & $3 \mathrm{yr}$ & Total & \\
\hline Intestinal obstruction & 1 & 1 & - & - & 2 & 1 & - & - & - & 1 & 0.861 \\
\hline Stenosis & 1 & & - & - & 1 & - & - & - & - & 0 & 0.945 \\
\hline Reflux & 1 & 1 & - & - & 2 & - & - & 1 & 1 & 2 & 0.261 \\
\hline Malabsorption & - & - & - & - & 0 & - & - & - & - & 0 & 1.000 \\
\hline Dumping & - & - & - & 1 & 1 & - & 1 & - & 1 & 2 & 0.861 \\
\hline Delayed emptying & 1 & - & 2 & - & 3 & - & - & - & - & 0 & 0.861 \\
\hline Other & 4 & - & 1 & - & 5 & 4 & - & - & - & 4 & 0.825 \\
\hline
\end{tabular}

A

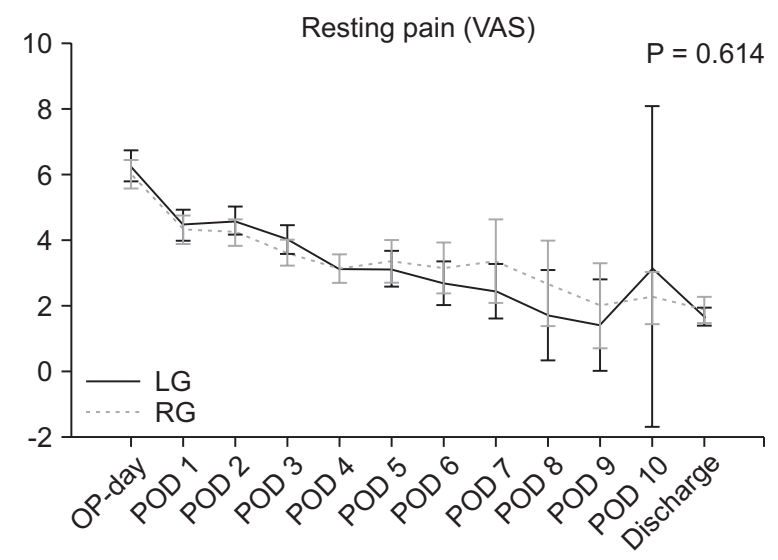

C

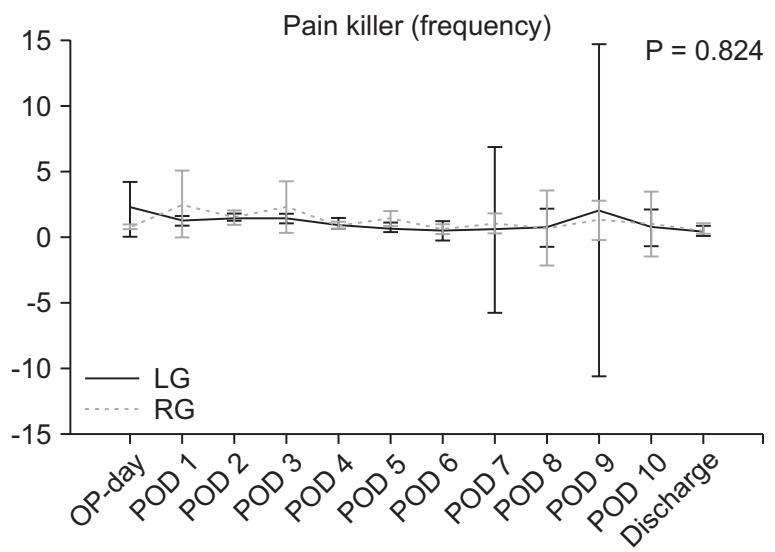

B

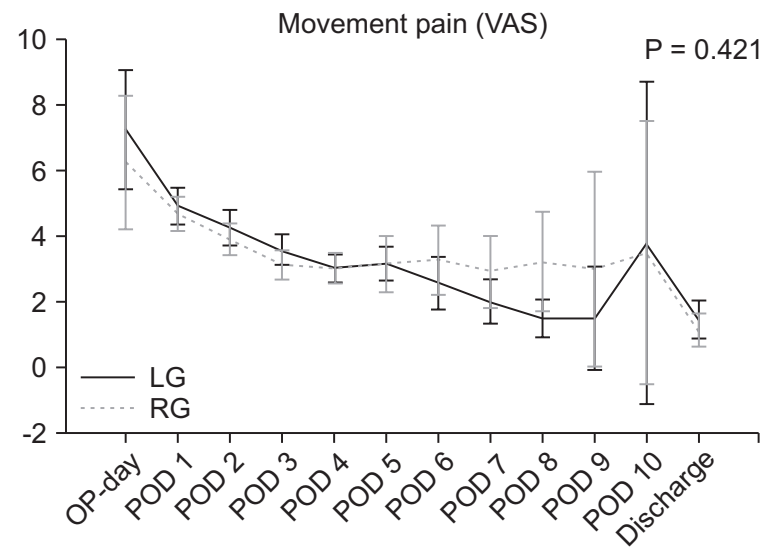

D 100

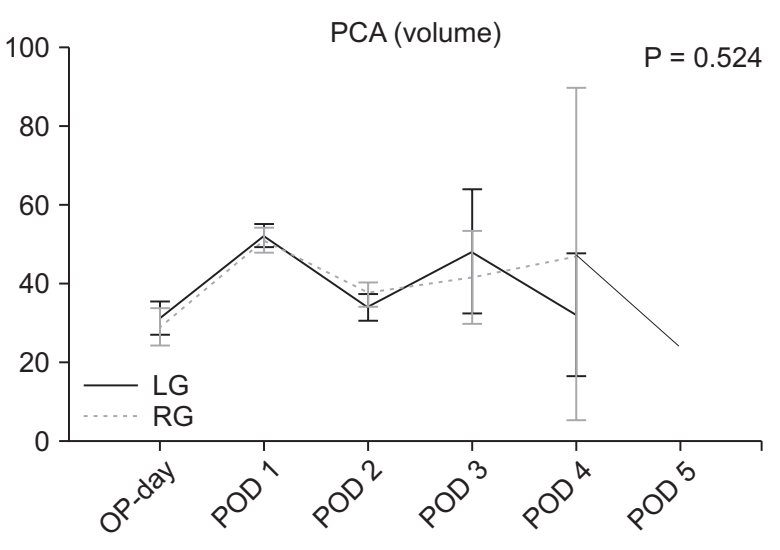

Fig. 3. Change of pain score and the use of pain killers. (A) Resting pain score from operation day (OP-day) to discharge $(\mathrm{P}=$ 0.614). (B) Movement pain score $(P=0.421$ ). (C) The use of painkiller (frequency, $P=0.824$ ). (D) The use of patient-controlled analgesia (PCA) (volume, $\mathrm{P}=0.524)$. VAS, visual analog scale; $L G$, laparoscopic gastrectomy; $R G$, robotic gastrectomy; POD, postoperative day. 
Better quality of life of men, distal gastrectomy, and stage II-III across several parameters

We compared TG and DG, PPG and DG, stage I and stage II-III, and male and female rather than a robotic and laparoscopic surgery from the initial data set (321 cases). A comparison between TG and DG was adjusted by PSM with 2 confounders, weight and the approach of the anastomosis. A comparison between PPG and DG was adjusted with weight and the approach of the anastomosis. A comparison between stage I and stage II-III was matched without a confounder. A comparison between male and female was matched with BMI as a confounder. DG showed better scores using 2 of C30 functioning scales, 6 of C30 symptom scales, and 6 of STO22 symptom scales than TG. DG was better in constipation but worse in emotional functioning and anxiety than PPG. Male patients showed better scores in general health status, 4 of C30 functioning scales, 7 of C30 symptom scales, and 6 of STO22 symptom scales than female patients. Stage II-III showed better scores at the 3 of C 30 functioning scales and 3 of C30 symptom scales (Table 3).

\section{DISCUSSION}

Robotic surgery is a procedure with greater use of technology. Recently, robotic surgery has attracted the attention of people with respect to big data and artificial intelligence. However, robotic surgery is more expensive than conventional laparoscopic surgery. Therefore, we need to determine the advantages that offset its high cost. Comparative studies have been conducted to analyze the oncological effect, complications,

Table 3. Analysis of the factors that cause the difference in quality of life (QOL)

\begin{tabular}{|c|c|c|c|c|c|}
\hline \multirow[b]{2}{*}{ QOL scale } & \multicolumn{5}{|c|}{ The group with a high score at an individual scale (P-value) } \\
\hline & $\begin{array}{l}\text { RG vs. } \mathrm{LG}^{\mathrm{a})} \\
(131 \text { vs. 131) }\end{array}$ & $\begin{array}{l}\text { TG vs. } \mathrm{DG}^{\mathrm{b})} \\
(53 \text { vs. } 53)\end{array}$ & $\begin{array}{c}\text { PPG vs. } \text { DG }^{\mathrm{c})} \\
(28 \text { vs. } 28)\end{array}$ & $\begin{array}{l}\text { Stage I vs. II-III } \\
\quad(49 \text { vs. } 49)\end{array}$ & $\begin{array}{c}\text { Male vs. female }^{\mathrm{e})} \\
\quad(124 \text { vs. 124) }\end{array}$ \\
\hline C30-General health status & NS $(0.402)$ & NS $(0.174)$ & NS (0.092) & NS $(0.126)$ & Male $(<0.001)$ \\
\hline C30-Physical functioning & NS $(0.577)$ & NS (0.659) & NS $(0.232)$ & NS $(0.120)$ & Male $(<0.001)$ \\
\hline C30-Role functioning & NS (0.209) & DG $(0.002)$ & NS (0.323) & Stage II-III (0.019) & Male $(<0.001)$ \\
\hline C30-Emotional functioning & NS (0.499) & NS (0.105) & PPG $(0.041)$ & Stage II-III $(<0.001)$ & Male $(<0.001)$ \\
\hline C30-Cognitive functioning & RG (0.005) & NS $(0.771)$ & NS (0.595) & Stage II-III (0.023) & Male $(<0.001)$ \\
\hline C30-Social functioning & NS (0.489) & DG $(0.027)$ & NS (0.817) & NS $(0.323)$ & NS (0.089) \\
\hline C30-Fatigue & NS (0.992) & TG $(0.017)$ & NS (0.758) & Stage I (0.041) & Female $(<0.001)$ \\
\hline C30-Nausea and vomiting & NS $(0.917)$ & TG $(0.034)$ & NS (0.994) & NS (0.775) & Female $(0.002)$ \\
\hline C30-Pain & NS (0.169) & TG $(0.010)$ & NS (0.909) & NS $(0.367)$ & Female $(<0.001)$ \\
\hline C30-Dyspnea & NS (0.526) & TG $(0.035)$ & NS $(0.771)$ & NS $(0.170)$ & Female $(0.040)$ \\
\hline C30-Insomnia & NS $(0.483)$ & NS $(0.873)$ & NS $(0.354)$ & Stage I (0.004) & Female $(<0.001)$ \\
\hline C30-Appetite loss & NS $(0.691)$ & NS (0.059) & NS (0.714) & Stage I (0.043) & Female (0.002) \\
\hline C30-Constipation & NS (0.829) & TG $(0.015)$ & PPG (0.037) & NS $(0.898)$ & Female $(<0.001)$ \\
\hline C30-Diarrhea & NS $(0.813)$ & NS $(0.247)$ & NS $(0.259)$ & NS $(0.287)$ & NS $(0.574)$ \\
\hline C30-Financial difficulties & NS $(0.371)$ & TG $(0.042)$ & NS (0.184) & NS $(0.825)$ & NS (0.396) \\
\hline STO22-Dysphagia & NS (0.195) & TG $(0.006)$ & NS (0.799) & NS $(0.434)$ & Female (0.005) \\
\hline STO22-Pain & NS (0.789) & TG $(0.003)$ & NS (0.966) & NS $(0.807)$ & Female $(<0.001)$ \\
\hline STO22-Reflux symptoms & NS $(0.097)$ & NS (0.144) & NS (0.509) & NS $(0.121)$ & Female $(<0.001)$ \\
\hline STO22-Eating restrictions & NS (0.638) & TG $(<0.001)$ & NS $(0.940)$ & NS $(0.347)$ & Female (0.004) \\
\hline STO22-Anxiety & NS $(0.957)$ & TG $(0.002)$ & DG (0.018) & NS $(0.817)$ & NS (0.093) \\
\hline STO22-Dry mouth & NS (0.793) & NS $(0.215)$ & NS (0.415) & NS $(0.300)$ & Female (0.018) \\
\hline STO22-Taste & NS $(0.848)$ & TG $(0.040)$ & NS $(0.263)$ & NS $(0.740)$ & NS $(0.459)$ \\
\hline STO22-Body image & NS (0.388) & TG $(0.008)$ & NS (0.327) & NS (0.089) & NS $(0.721)$ \\
\hline STO22-Hair loss & NS $(0.101)$ & NS $(0.631)$ & NS (0.747) & NS (0.117) & Female (0.008) \\
\hline
\end{tabular}

The QOL questionnaire (QLQ)-C30 and QLQ-STO22 developed by the European Organization for Research and Treatment of Cancer were used for the QOL survey.

RG, robotic gastrectomy; LG, laparoscopic gastrectomy; TG, total gastrectomy; DG, distal gastrectomy; PPG, pylorus-preserving gastrectomy; NS, not significant $(P \geq 0.05)$.

${ }^{a}$ A comparison between RG and LG was adjusted by propensity score matching with age, American Society of Anesthesiologists physical status classification, and pathologic TNM (174 vs. $147 \rightarrow 131$ vs. 131). ${ }^{b}$ A comparison between TG and DG was matched

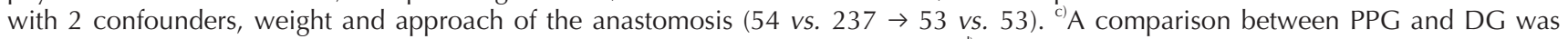
matched with weight and approach of the anastomosis (28 vs. $237 \rightarrow 28$ vs. 28). ${ }^{\text {d) }}$ A comparison between stage I and stage II-III was matched without a confounder (272 vs. $49 \rightarrow 49$ vs. 49). ${ }^{\text {e) }}$ A comparison between male and female patients was matched with body mass index (190 vs. $131 \rightarrow 124$ vs. 124). 
and surgical performance (e.g., the amount of bleeding, length of hospital stay, and operation time) of robotic surgery [16]. Until now, it does not appear to be superior to conventional laparoscopic surgery for gastric cancer $[12,17]$.

In this study, robotic surgery was different from laparoscopic surgery only in the C30 cognitive functioning scale. This resulted from the difference in preoperative baseline measurements. For budget issues, this study could not randomize the robotic and laparoscopic groups. The cognitive functioning scale may be related to the economic background of patients who chose robotic surgery. Comparing the change before and after surgery, there was no difference noticed in QOL including cognitive functioning between robotic and laparoscopic surgery. Since the technology applied to robotic surgery has been upgraded continuously, the benefit for gastric cancer might be discovered in the future.

It is difficult to define an overall indicator by integrating individual scales, and it is difficult to clarify the cause-andeffect relationship. Nevertheless, the value of this study is that we tried a prospective approach to control the variables associated with QOL. This is the only prospective study that verified the QOL of robotic surgery for gastric cancer. Similar prospective studies of QOL were performed to compare open surgery and laparoscopic surgery or to compare the type of anastomosis [18].

In this study, DG received a good score on 14 items when compared to TG. That is consistent with the results from previous studies comparing DG and TG [7,19-22]. In fact, patients who underwent TG complained about weight loss and frequent bowel movement. In this study, the scores of nausea/ vomiting, constipation, dysphagia, and eating restrictions of TG patients were found to be poor. Referring to an article published in 2016, the QOL comparison of TG and DG in our study is similar to theirs by 18 scales of 24 [22]. This study was derived from an earlier study comparing robotic surgery and laparoscopic surgery. There are limits to the population for analyzing these other factors, though.

We found 3 articles comparing QOL with the stage of gastric cancer. In one article, there was no significant difference in the QOL between stage I/II and III/IV [23]. In another article, there was no difference between the patients who underwent adjuvant chemotherapy and those who did not undergo adjuvant chemotherapy [24]. Nevertheless, another study published in 2018 showed that the role functioning scale and

Table 4. Chronological change of quality of life (QOL): compare of 4 study groups

\begin{tabular}{|c|c|c|c|c|c|}
\hline QOL scale & This study & Ref. 7 & Ref. 29 & Ref. 30 & Concordance (3/4) \\
\hline C30-General health status & $\pi$ & $\pi$ & $\pi$ & $\pi$ & $\pi$ \\
\hline C30-Physical functioning & $\mathfrak{1}$ & $\mathfrak{1}$ & $\uparrow$ & $\uparrow$ & $\uparrow$ \\
\hline C30-Role functioning & $\pi$ & 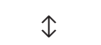 & $\uparrow$ & $\uparrow$ & $\mathfrak{1}$ \\
\hline C30-Emotional functioning & $\pi$ & $\pi$ & $\pi$ & $\pi$ & $\pi$ \\
\hline C30-Cognitive functioning & $\uparrow$ & $\pi$ & $\uparrow$ &  & ? \\
\hline C30-Social functioning & $\pi$ & $\pi$ & $?$ & $\pi$ & $\pi$ \\
\hline C30-Fatigue & $\searrow$ & $\uparrow$ & 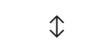 & $\uparrow$ & $\uparrow$ \\
\hline C30-Nausea and vomiting & $\uparrow$ & $\uparrow$ & $\uparrow$ & $\uparrow$ & $\uparrow$ \\
\hline C30-Pain & $\mathfrak{1}$ & $\uparrow$ & 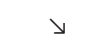 & $\mathfrak{1}$ & $\uparrow$ \\
\hline C30-Dyspnea & $\searrow$ & $\uparrow$ & $\uparrow$ & $\uparrow$ & $\uparrow$ \\
\hline C30-Insomnia & $\searrow$ & $\uparrow$ & $?$ & $?$ & $?$ \\
\hline C30-Appetite loss & $\searrow$ & $\uparrow$ & $\uparrow$ & $\uparrow$ & $\mathfrak{1}$ \\
\hline C30-Constipation &  & $\uparrow$ & $\uparrow$ & $?$ & $?$ \\
\hline C30-Diarrhea & $\uparrow$ & $\uparrow$ & $\uparrow$ & $\uparrow$ & $\uparrow$ \\
\hline C30-Financial difficulties & $\downarrow$ & $\searrow$ & $\searrow$ & $\searrow$ & $\searrow$ \\
\hline STO22-Dysphagia & $\uparrow$ & $\uparrow$ & $\uparrow$ & $\uparrow$ & $\uparrow$ \\
\hline STO22-Pain & $\uparrow$ & $\searrow$ & $\uparrow$ & $\uparrow$ & $\uparrow$ \\
\hline STO22-Reflux symptoms & $\uparrow$ & $\uparrow$ & $?$ & $\uparrow$ & $\uparrow$ \\
\hline STO22-Eating restrictions & $\uparrow$ & $\uparrow$ &  & $\uparrow$ & $\uparrow$ \\
\hline STO22-Anxiety & $\uparrow$ & $\searrow$ & $\uparrow$ & $?$ & $?$ \\
\hline STO22-Dry mouth & $\mathfrak{1}$ & 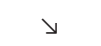 & 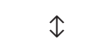 & $\uparrow$ & $\uparrow$ \\
\hline STO22-Taste & $\uparrow$ & $\uparrow$ & $\uparrow$ & $\uparrow$ & $\uparrow$ \\
\hline STO22-Body image & $\uparrow$ & $\uparrow$ & $\uparrow$ & $\uparrow$ & $\uparrow$ \\
\hline STO22-Hair loss & $\uparrow$ & $?$ & $\uparrow$ & $\uparrow$ & $\uparrow$ \\
\hline
\end{tabular}

The QOL questionnaire (QLQ)-C30 and QLQ-STO22 developed by the European Organization for Research and Treatment of Cancer were used for the QOL survey.

Ref, reference; $\downarrow$, recovery (function scale decreased and partially recovered, symptom scale increased and partially recovered); $\lambda$, increase (function scale finally over baseline); $\triangleleft$, decrease (symptom scale finally under baseline). 
reflux scale were good in the advanced stage (>stage II); that is similar to our analysis [25]. It seems that the experience of survival from cancer might change the threshold of an individual's satisfaction.

We found that female patients had poor scores on many indicators; physical functioning, fatigue, nausea/vomiting, insomnia, appetite loss, diarrhea, dysphagia, pain, eating restrictions, anxiety, taste, and hair loss. Referring to the previous study, the QOL of the general population seems to be sexually biased [26]. Referring to a well-designed study that compared QOL before and after coronary artery bypass surgery, they concluded that the baseline level of QOL and the change of QOL by surgery differed according to sex [27,28]. Interestingly, an article about gastric surgery presented results similar to ours in terms of sex differences [25]. Therefore, our clinicians might keep in mind that the ratio of males and females affects the outcomes of the QOL study.

We examined the chronological changes of QOL for this study. We found 3 articles that described the chronological change of the QOL after gastrectomy; these studies were performed in Korea and Japan [7,29,30]. We analyzed the line graph about QOL scales from these articles and arranged them in 3 categories as follows: (1) 'recovery ( $\$$ )' - functioning scale decreased and partially recovered, or symptom scale increased and partially recovered; (2) 'increase (ㄱ)' - function scale finally increased over baseline; (3) 'decrease ( $\$ )' - symptom scale finally decreased under the baseline (Table 4). We defined 'concordance' of a scale when 3 of 4 articles showed the same pattern of the scale. Therefore, C30-general health status, 4 of C30 functioning scales, 7 of C30 symptom scales, and 8 of STO22 symptom scales showed concordance among the 4 articles. The results demonstrate the reliability or reproducibility of the QOL study. Patients are afraid of changes before and after surgery. By predicting the chronological changes of the QOL profile, patients will be free from fear and find a solution easily to their problem.

In conclusion, robotic and laparoscopic approaches for gastric cancer surgery did not differ regarding QOL, except in the C30 cognitive functioning scale. Male (vs. female), DG (vs. TG), and stage II-III (vs. stage I) resulted in better QOL among the several parameters chosen for the study.

\section{ACKNOWLEDGEMENTS}

\section{Fund/Grant Support}

This study was supported by a grant from the National $R \& D$ Program for Cancer Control, Ministry of Health \& Welfare, Republic of Korea (1020410).

\section{Conflict of interest}

No potential conflict of interest relevant to this article was reported.

\section{ORCID iD}

Jong-Ho Choi: https://orcid.org/0000-0001-6963-7075

Sang-Uk Han: https://orcid.org/0000-0001-5615-4162

Han-Kwang Yang: https://orcid.org/0000-0003-3495-3048

Young-Woo Kim: https://orcid.org/0000-0002-1559-9672

Keun Won Ryu: https://orcid.org/0000-0002-5935-9777

Joong-Min Park: https://orcid.org/0000-0002-8582-6391

Ji Yeong An: https://orcid.org/0000-0003-1690-4947

Min-Chan Kim: https://orcid.org/0000-0003-1905-3316

Sungsoo Park: https://orcid.org/0000-0002-1779-8683

Kyo Young Song: https://orcid.org/0000-0002-5840-1638

Sung Jin Oh: https://orcid.org/0000-0002-2155-1376

Seong-Ho Kong: https://orcid.org/0000-0002-3929-796X

Byoung Jo Suh: https://orcid.org/0000-0003-4425-4822

Dae Hyun Yang: https://orcid.org/0000-0002-0679-1435

Tae Kyung Ha: https://orcid.org/0000-0001-7320-5507

Hyoung-Il Kim: https://orcid.org/0000-0002-6134-4523

Woo Jin Hyung: https://orcid.org/0000-0002-8593-9214

Hyuk-Joon Lee: https://orcid.org/0000-0002-9530-647X

\section{Author Contribution}

Conceptualization: HJL, WJH, SUH, MCK, SP

Formal Analysis: JHC, HKY, HJL, SHK

Investigation: YWK, KWR, JMP, JYA, KYS, SJO, BJS, DHY, TKH, HIK

Methodology: HJL, WJH, HIK

Project Administration: HJL

Writing - Original Draft: JHC, HJL

Writing - Review \& Editing: SUH, HKY, YWK, KWR, JMP, JYA, MCK, SP, KYS, SJO, SHK, BJS, DHY, TKH, HIK, WJH

\section{REFERENCES}

1. Lee HJ, Yang HK. Laparoscopic gastrectomy for gastric cancer. Dig Surg 2013;30: $132-41$.

2. Kitano S, Shiraishi N, Uyama I, Sugihara
K, Tanigawa N; Japanese Laparoscopic Surgery Study Group. A multicenter study on oncologic outcome of laparoscopic gastrectomy for early cancer in Japan.
Ann Surg 2007:245:68-72.

3. Kim HH, Han SU, Kim MC, Kim W, Lee HJ, Ryu SW, et al. Effect of laparoscopic distal gastrectomy vs open distal 
gastrectomy on long-term survival among patients with stage I gastric cancer: the KLASS-01 randomized clinical trial. JAMA Oncol 2019:5:506-13.

4. Oh SY, Lee HJ, Yang HK. Pyloruspreserving gastrectomy for gastric cancer. J Gastric Cancer 2016;16:63-71.

5. Jung DH, Ahn SH, Park DJ, Kim HH. Proximal gastrectomy for gastric cancer. J Gastric Cancer 2015;15:77-86.

6. Choi JH, Suh YS, Park SH, Kong SH, Lee HJ, Kim WH, et al. Risk factors of microscopic invasion in early gastric cancer. J Gastric Cancer 2017;17:331-41.

7. Kobayashi D, Kodera Y, Fujiwara M, Koike M, Nakayama G, Nakao A. Assessment of quality of life after gastrectomy using EORTC QLQ-C30 and STO22. World J Surg 2011:35:357-64.

8. Yun YH, Park YS, Lee ES, Bang SM, Heo DS, Park SY, et al. Validation of the Korean version of the EORTC QLQ-C30. Qual Life Res 2004;13:863-8.

9. Lee JH, Lee HJ, Choi YS, Kim TH, Huh YJ, Suh YS, et al. Postoperative quality of life after total gastrectomy compared with partial gastrectomy: longitudinal evaluation by European Organization for Research and Treatment of Cancer-OG25 and STO22. J Gastric Cancer 2016;16:2309.

10. Terashima M, Tokunaga M, Tanizawa Y, Bando E, Kawamura T, Miki Y, et al. Robotic surgery for gastric cancer. Gastric Cancer 2015;18:449-57.

11. Han DS, Suh YS, Ahn HS, Kong SH, Lee HJ, Kim WH, et al. Comparison of surgical outcomes of robot-assisted and laparoscopy-assisted pylorus-preserving gastrectomy for gastric cancer: a propensity score matching analysis. Ann Surg Oncol 2015:22:2323-8.

12. Kim HI, Han SU, Yang HK, Kim YW, Lee $\mathrm{HJ}$, Ryu KW, et al. Multicenter prospective comparative study of robotic versus laparoscopic gastrectomy for gastric adenocarcinoma. Ann Surg 2016;263:1039.

13. Japanese Gastric Cancer Association.
Japanese gastric cancer treatment guidelines 2010 (ver. 3). Gastric Cancer 2011;14:113-23.

14. Sakuramoto S, Sasako M, Yamaguchi T, Kinoshita T, Fujii M, Nashimoto A, et al. Adjuvant chemotherapy for gastric cancer with S-1, an oral fluoropyrimidine. N Engl J Med 2007;357:1810-20.

15. Noh SH, Park SR, Yang HK, Chung HC, Chung IJ, Kim SW, et al. Adjuvant capecitabine plus oxaliplatin for gastric cancer after D2 gastrectomy (CLASSIC): 5-year follow-up of an open-label, randomised phase 3 trial. Lancet Oncol 2014:15:1389-96.

16. Lee EK, Park E, Oh WO, Shin NM. Comparison of the outcomes of robotic cholecystectomy and laparoscopic cholecystectomy. Ann Surg Treat Res 2017;93:27-34.

17. Park JM, Kim HI, Han SU, Yang HK, Kim YW, Lee HJ, et al. Who may benefit from robotic gastrectomy?: a subgroup analysis of multicenter prospective comparative study data on robotic versus laparoscopic gastrectomy. Eur J Surg Oncol 2016:42:1944-9.

18. Straatman J, van der Wielen N, Joosten PJ, Terwee CB, Cuesta MA, Jansma EP, et al. Assessment of patient-reported outcome measures in the surgical treatment of patients with gastric cancer. Surg Endosc 2016:30:1920-9.

19. Jentschura D, Winkler M, Strohmeier N, Rumstadt B, Hagmüller E. Qualityof-life after curative surgery for gastric cancer: a comparison between total gastrectomy and subtotal gastric resection. Hepatogastroenterology 1997:44:1137-42.

20. Goh YM, Gillespie C, Couper G, PatersonBrown S. Quality of life after total and subtotal gastrectomy for gastric carcinoma. Surgeon 2015;13:267-70.

21. Isozaki H, Matsumoto S, Murakami $\mathrm{S}$, Takama T, Sho T, Ishihara K, et al. Diminished gastric resection preserves better quality of life in patients with early gastric cancer. Acta Med Okayama 2016; 70:119-30.

22. Lee SS, Chung HY, Kwon OK, Yu W. Long- term quality of life after distal subtotal and total gastrectomy: symptom- and behavior-oriented consequences. Ann Surg 2016:263:738-44.

23. Huang CC, Lien HH, Wang PC, Yang JC, Cheng CY, Huang CS. Quality of life in disease-free gastric adenocarcinoma survivors: impacts of clinical stages and reconstructive surgical procedures. Dig Surg 2007:24:59-65.

24. Bae JM, Kim S, Kim YW, Ryu KW, Lee $\mathrm{JH}$, Noh JH, et al. Health-related quality of life among disease-free stomach cancer survivors in Korea. Qual Life Res 2006;15:1587-96.

25. Brenkman HJ, Tegels JJ, Ruurda JP, Luyer MD, Kouwenhoven EA, Draaisma WA, et al. Factors influencing health-related quality of life after gastrectomy for cancer. Gastric Cancer 2018;21:524-32.

26. Nolte S, Liegl G, Petersen MA, Aaronson NK, Costantini A, Fayers PM, et al. General population normative data for the EORTC QLQ-C30 health-related quality of life questionnaire based on 15,386 persons across 13 European countries, Canada and the Unites States. Eur J Cancer 2019;107:153-63.

27. Peric V, Borzanovic M, Stolic R, Jovanovic A, Sovtic S, Djikic D, et al. Quality of life in patients related to gender differences before and after coronary artery bypass surgery. Interact Cardiovasc Thorac Surg 2010;10:232-8.

28. Sjöland H, Wiklund I, Caidahl K, Hartford M, Karlsson T, Herlitz J. Improvement in quality of life differs between women and men after coronary artery bypass surgery. J Intern Med 1999:245:445-54.

29. Yu W, Park KB, Chung HY, Kwon OK, Lee SS. Chronological changes of quality of life in long-term survivors after gastrectomy for gastric cancer. Cancer Res Treat 2016;48:1030-6.

30. Kim AR, Cho J, Hsu YJ, Choi MG, Noh JH, Sohn TS, et al. Changes of quality of life in gastric cancer patients after curative resection: a longitudinal cohort study in Korea. Ann Surg 2012;256:1008-13. 